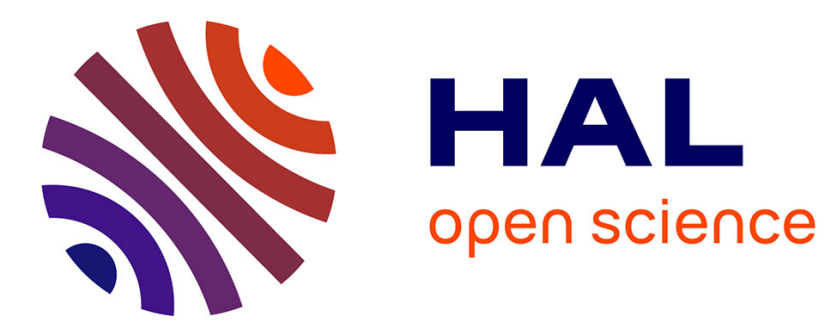

\title{
Synthesis of Orthogonally N-Protected, C-4 Functionalized Cyclic Guanidines from L-Serine
}

Diego Silva, Edmilson Maria, Rosa Suárez Ordóñez, Josiane Thierry, Kevin Cariou, Robert Dodd

\section{- To cite this version:}

Diego Silva, Edmilson Maria, Rosa Suárez Ordóñez, Josiane Thierry, Kevin Cariou, et al.. Synthesis of Orthogonally N-Protected, C-4 Functionalized Cyclic Guanidines from L-Serine. SYNLETT, 2017, 28 (07), pp.815-818. 10.1055/s-0036-1589935 . hal-02307213

\section{HAL Id: hal-02307213 \\ https://hal.science/hal-02307213}

Submitted on 7 Oct 2019

HAL is a multi-disciplinary open access archive for the deposit and dissemination of scientific research documents, whether they are published or not. The documents may come from teaching and research institutions in France or abroad, or from public or private research centers.
L'archive ouverte pluridisciplinaire HAL, est destinée au dépôt et à la diffusion de documents scientifiques de niveau recherche, publiés ou non, émanant des établissements d'enseignement et de recherche français ou étrangers, des laboratoires publics ou privés. 


\section{Synthesis of Orthogonally N-Protected, C-4 Functionalized Cyclic Guanidines from L-Serine}

\author{
Diego R. C. Silva, ${ }^{\mathrm{a}, \mathrm{b}}$ \\ Edmilson J. Maria, ${ }^{\text {b }}$ \\ Rosa M. Suárez Ordóñez,a \\ Josiane Thierry, ${ }^{a}$ \\ Kevin Cariou, ${ }^{* a}$ \\ Robert H. Dodd*a
}

a Institut de Chimie des Substances Naturelles, CNRS UPR 2301, Univ. Paris-Sud, Univ. ParisSaclay, 1 Avenue de la Terrasse, 91198 Gif-surYvette, France

b Universidade Estadual do Norte Fluminense Darcy Ribeiro, Campos dos Goytacazes, RJ, Brazil

*Kevin.cariou@cnrs.fr, * Robert.dodd@cnrs.fr

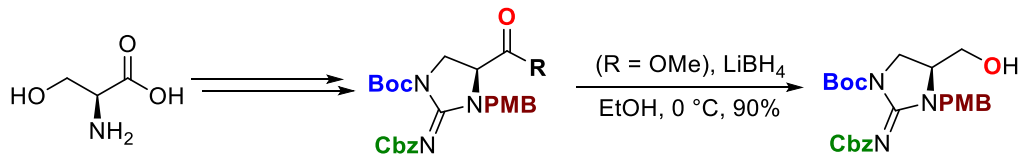

L-serine

$\mathbf{R}=\mathrm{N}(\mathrm{Me}) \mathrm{OMe}, 13$ steps, $24 \%$

orthogonally protected

\begin{abstract}
Received: DOI:

Abstract A straightforward and efficient preparation of 5 membered cyclic guanidines bearing an ester, a hydroxymethyl or a Weinreb amide functional group at C-4 is described from L-serine. The novel synthetic route provides cyclic guanidines in which the three nitrogen atoms are orthogonally protected making them highly suitable for further transformations into natural products or their analogues via the introduced functional groups.
\end{abstract}

Key words cyclic guanidine, amino acid, azide, orthogonal protection, Weinreb amide

The cyclic guanidine motif is present in a wide range of naturally-occurring compounds, ${ }^{1}$ notably as a structural unit of non-proteinogenic amino acids, examples of which are enduracididine (1), ${ }^{2}$ its 3-hydroxy derivatives (2), capreomycidine ${ }^{4}(3)$ and tetrahydrolathyrin ${ }^{5}(4)$ or of alkaloids such as trachycladindole $\mathrm{B}^{6}(5)$ and its derivatives (Figure 1). These amino acids have been isolated either in their free form or as components of cyclic peptides. Thus, compound $\mathbf{1}$ is a structural unit of the cyclopeptide enduracidin ${ }^{2}$ and of the recently described teixobactin, ${ }^{7}$ compound $\mathbf{2}$ of the mannopeptimycins ${ }^{3}$ and compound $\mathbf{3}$ of capreomycins and tuberactinomycins, ${ }^{8}$ all of which display antibiotic or antifungal action. In terms of synthesis, formation of the cyclic guanidine unit usually depends on the generation of a 1,2- or 1,3-diamino motif which classically can then be cyclized using cyanogen bromide, an inexpensive but highly toxic reagent. Alternatively, one of the amino groups can be coupled to a reactive isothiourea derivative which then undergoes cyclization upon heating. ${ }^{9}$ Starting from a 1-hydroxyl-2-(or 3-)-guanidine unit, a Mitsunobu-type cyclodehydration has also been used to prepare a 5-(or 6-) membered cyclic guanidine. 10-12

We ourselves have recently described the syntheses of both Lenduracididine (1) ${ }^{13}$ and tetrahydrolathyrin (4) ${ }^{14}$ by way of prior iminoiodane-mediated aziridination of a common, easily accessible starting material, L-allylglycine. Subsequent nucleophilic opening of the aziridine by azide (for enduracididine) or nitrile (for tetrahydrolathyrin) provided the required diamino precursors after reduction. The lack of diasteoselectivity in the aziridination step was, however, a complicating factor in both cases.

(

Figure 1 Examples of natural products containing a C-4 substituted cyclic guanidine structural unit

It occurred to us that a more convergent way of synthesizing cyclic guanidine-containing compounds, be they natural products, analogues thereof or in the context of drug discovery, would consist of preparing a pre-formed cyclic guanidine moiety bearing a functional group which could thereafter be used to link another molecule (e.g., an amino acid, an indole...). Such a tactic was recently described by us whereby halogenated cyclic guanidines were obtained in one step by halocyclization of unsaturated guanidines using a combination of lithium halides and Koser's reagent. ${ }^{15}$ We now report herein the efficient preparation, starting from L-serine, of 5-membered cyclic guanidines functionalized at C-4 with an ester, a Weinreb amide or a hydroxymethyl group and in which, moreover, the three guanidine nitrogen atoms are orthogonally protected. The possibility of accessing a mono- $N$-deprotected cyclic guanidine could be highly valuable, for example, for the introduction of different substituents on each nitrogen atom in the context of a structure-activity relationship study of a particular bio-active molecule. 
The success of our methodology was very much dependent on the judicious choice of nitrogen atom protecting groups. ${ }^{16}$ Indeed, a frequently encountered problem in working with partially $N$-protected cyclic guanidines is scrambling of the protecting group $(\mathrm{s})^{17}$ making characterization and purification of products difficult and lowering yields. For our initial studies starting from L-serine methyl ester (6), we thus chose to protect the amine function with a $p$-methoxybenzyl (PMB) group not prone to migration but easily removed under mild oxidizing conditions (Scheme 1). Additionally, the electron-rich nature of the group should assist in the subsequent cyclization step. Thus, reaction of 6 with $p$-methoxybenzaldehyde (7) in methanol in the presence of triethylamine followed by sodium borohydridepromoted reduction of the resulting intermediate imine provided the desired $N$-PMB serine methyl ester 8 in $81 \%$ overall yield. Compound $\mathbf{8}$ in dichloromethane was then treated at $-78{ }^{\circ} \mathrm{C}$ with thionyl chloride in the presence of pyridine to give the oxathiazolidine derivative 9 . The latter was oxidized to the cyclic sulfamidate 10 in $77 \%$ overall yield using catalytic ruthenium trichloride and sodium metaperiodate in acetonitrile. ${ }^{18}$ Regioselective ring-opening of compound $\mathbf{1 0}$ in acetone was very efficiently achieved by the action of aqueous sodium azide (Warning!) at room temperature, furnishing methyl (2S)-3-azido-2-(p-methoxybenzyl)aminopropanoate 11 in $85 \%$ yield. ${ }^{19}$

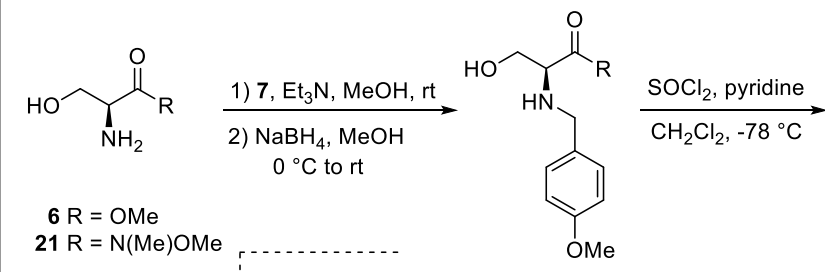

$$
\begin{aligned}
& 21 \mathrm{R}=\mathrm{N}(\mathrm{Me}) \mathrm{OMe} \\
& \begin{aligned}
8 \mathrm{R} & =\mathrm{OMe} 81 \% \\
72 \mathrm{R} & =\mathrm{N}(\mathrm{Me}) \mathrm{OMe} 91 \%
\end{aligned}
\end{aligned}
$$

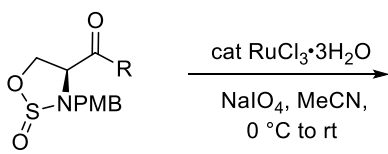

$$
\begin{aligned}
& 9 \mathrm{R}=\mathrm{OMe} \\
& 23 \mathrm{R}=\mathrm{N}(\mathrm{Me}) \mathrm{OMe} \\
& \mathrm{PMB}=p \text {-methoxybenzyl } \\
& 24 \text { or } \frac{\text { 1) } \mathrm{NaN}_{3}, \text { acetone/ } \mathrm{H}_{2} \mathrm{O}, \mathrm{rt}}{2} \underset{\mathrm{H}_{2} \mathrm{SO}_{4}, \mathrm{CH}_{2} \mathrm{Cl}_{2}, \mathrm{rt}}{\longrightarrow} \\
& 11 \mathrm{R}=\mathrm{OMe} 85 \% \\
& 25 \mathrm{R}=\mathrm{N}(\mathrm{Me}) \mathrm{OMe} 68 \%
\end{aligned}
$$

Scheme 1 Preparation of the 2-amino-3-azido precursors for cyclic guanidine formation

Transformation of azido derivative 11 into the orthogonally $\mathrm{N}$ protected cyclic guanidine 4-carboxylic ester $\mathbf{1 6}$ is depicted in Scheme 2. Thus, the azido function of $\mathbf{1 1}$ was first reduced to the primary amine $\mathbf{1 2}$ via a Staudinger reaction using triphenylphosphine in aqueous dichloromethane. This amine was not purified but was reacted directly with 1,3bis(benzyloxycarbonyl)-2-methylisothiourea (13) in the presence of triethylamine in dichloromethane. ${ }^{20}$ The resulting guanidino derivative 14, obtained in 95\% overall yield for both steps, underwent cyclization when heated for 2 days at $70{ }^{\circ} \mathrm{C}$ in acetonitrile, providing a $75 \%$ yield of methyl 2iminoimidazolidine-4-carboxylate 15. ${ }^{21}$ Finally, treatment of compound 15 with excess Boc anhydride and DMAP in acetonitrile led to the targeted fully $N$-protected, C-4 functionalized cyclic guanidine 16 in 73\% yield. The latter could furthermore be transformed in $90 \%$ yield to the C-4 hydroxymethyl derivative $\mathbf{1 7}$ by reduction of the ester with lithium borohydride in ethanol at $0{ }^{\circ} \mathrm{C}$. The orthogonality of our design was illustrated by the selective deprotection of the $\mathrm{Cbz}$ of $\mathbf{1 6}$ to give 18 by hydrogenolysis. Thus it is possible to go from 2,3 diprotected guanidine (15) to tri-protected (16), to 1,3 diprotected guanidine (18).

$$
\text { Schements }
$$

For the preparation of the Weinreb amide analogue of the cyclic guanidine ester 16, the corresponding amide of L-serine (i.e., compound 21) was first synthesized as shown in Scheme 3. This two-step procedure consisted of EDCI-promoted coupling of commercial $\mathrm{N}$-Boc-L-serine (19) with $\mathrm{N}, \mathrm{O}$ dimethylhydroxylamine in the presence of $N$-methylmorpholine (NMM) to give amide 20. Removal of the $\mathrm{N}$-Boc protecting group using trifluoroacetic acid in dichloromethane then provided $\mathbf{2 1}$ in $72 \%$ overall yield.

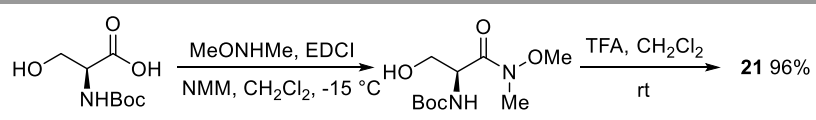

$$
\begin{aligned}
& 19 \\
& 2075 \%
\end{aligned}
$$

Scheme 3 Preparation of the Weinreb amide of L-serine

Application to $\mathbf{2 1}$ of the same reaction sequence used for the preparation of cyclic guanidine $\mathbf{1 6}$ from L-serine methyl ester (6) then allowed efficient access to optically pure, $N$-protected cyclic guanidine derivative $\mathbf{2 9}$ having now a Weinreb amide at 
the C-4 position suitable for further transformations (Schemes 1 and 2). ${ }^{20,21}$

In conclusion, esters 15, 16 or 18 primary alcohol 17 and Weinreb amide $\mathbf{2 9}$ represent five functionalized and stereochemically defined cyclic guanidine derivatives which can serve as starting materials for the synthesis of a variety of natural products containing this 5-membered heterocyclic unit. An added feature of the synthetic strategy presented here is that these key compounds can be obtained with each nitrogen atom of the guanidine unit being orthogonally protected allowing their selective deprotection and substitution in the context of a structure-activity study (e.g., $N$-alkylation as in the trachycladindoles 5) or of total synthesis (e.g., the mannopeptimycins). ${ }^{10 \mathrm{~b}}$ Such transformations are the object of our ongoing studies.

\section{Acknowledgment}

The authors are grateful to Conselho Nacional de Desenvolvimento Científico e Tecnológico (CNPq, Brazil) for financial support and D.R.C.S is thankful to CAPES, Brazil for a scholarship. We thank the Institut de Chimie des Substances Naturelles for financial and technical support.

\section{Supporting Information}

YES (this text will be updated with links prior to publication)

\section{Primary Data}

NO (this text will be deleted prior to publication)

\section{References and Notes}

(1) (a) Berlinck, R. G. S. Nat. Prod. Rep. 1996, 13, 377. (b) Berlinck, R. G. S.; Burtoloso, A. C. B.; Kossuga, M. H. Nat. Prod. Rep. 2008, 25, 919. (c) Berlinck, R. G. S.; Burtoloso, A. C. B.; Trindade-Silva, A. E.; Romminger, S.; Morais, R. P.; Bandeira, K.; Mizuno C. M. Nat. Prod. Rep. 2010, 27, 1871. (d) Castagnolo, D.; Schenone, S.; Botta, M. Chem. Rev. 2011, 111, 5247. (e) Berlinck, R. G. S.; Trindade-Silva A. E.; Santos, M. F. C. Nat. Prod. Rep. 2012, 29, 1382.

(2) (a) Hori, S.; Kameda, Y. J. Antibiot. 1968, 21, 665. (b) Higashide, E.; Hatano, K.; Shibata, M.; Nakazawa, K. J. Antibiot. 1968, 21, 126. (c) Asai, M.; Muroi, M.; Sugita, N.; Kawashima, H.; Mizuno, K.; Miyake, A. J. Antibiot. 1968, 21, 138.

(3) He, H.; Williamson, R. T.; Shen, B.; Graziani, E. I.; Yang, H. Y.; Sakya, S. M.; Petersen, P. J.; Carter, G. T. J. Am. Chem. Soc. 2002, 124, 9729.

(4) Herr, E. B.; Haney, M. E.; Pittenger, G. E.; Higgins, C. E. Proc. Ind. Acad. Sci. 1960, 69, 134.

(5) Fellows, L. E.; Bell, E. A.; Lee, T. S.; Janzen, D. H. Phytochemistry 1979, 18, 1333.

(6) Capon, R. J.; Peng, C.; Dooms, C. Org. Biomol. Chem. 2008, 6, 2765 2771.

(7) Ling, L. L.; Schneider, T.; Peoples, A. J.; Spoering, A. L.; Engels, I.; Conlon, B. P.; Mueller, A.; Schäberle, T. F.; Hughes, D. E.; Epstein, S.; Jones, M.; Lazarides, L.; Steadman,V. A.; Cohen, D. R.; Felix, C. R.; Fetterman, K. A.; Millett, W. P.; Nitti, A. G.; Zullo, A. M.; Chen, C.; Lewis, K. Nature 2015, 517, 455.

(8) (a) Nomoto, S.; Teshima, T.; Wakamiya, T.; Shiba, T. Tetrahedron 1978, 34, 921. (b) Yoshioka, H.; Aoki, T.; Goko, H.; Nakatsu, K.; Noda, T.; Sakakibara, H.; Take, T.; Nagata, A.; Abe, J. Tetrahedron Lett. 1971, 23, 2043.

(9) Su, W. Synth. Commun. 1996, 26, 407

(10) (a) Schwörer, C. J.; Oberthür, M. Eur. J. Org. Chem. 2009, 6129. (b) Olivier, K. S.; Van Nieuwenhze, M. S. Org. Lett. 2010, 12, 1680.
(11) For other leading references dealing with the synthesis of cyclic guanidine amino acids, see: (a) Fischer, S. N.; Schwörer, C. J.; Oberthür, M. Synthesis 2014, 46, 2234. (b) Fuse, S.; Koinuma, H.; Kimbara, A.; Izumikawa, M.; Mifune, Y.; He, H.; Shin-Ya, K.; Takahashi, T.; Doi, T. J. Am. Chem. Soc. 2014, 136, 12011. (c) Moeschwitzer, V. D.; Kariuki, B. M.; Redman, J. E. Tetrahedron Lett. 2013, 54, 4526. (d) Craig, W.; Chen, J.; Richardson, D.; Thorpe, R.; Yuan, Y. Org. Lett. 2015, 17, 4620. (e) Giltrap, A. M.; Dowman, L. J.; Nagalingam, G.; Ochoa, J. L.; Linington, R. G.; Britton, W. J.; Payne, R. J. Org. Lett. 2016, 18, 2788. (f) Wang, B.; Liu, Y.; Jiao, R.; Feng, Y.; Li, Q.; Chen, C.; Liu, L.; He, G.; Chen, G. J. Am. Chem. Soc. 2016, $138,3926$.

(12) DeMong, D. E.; Williams, R. M. Tetrahedron Lett. 2001, 42, 3529.

(13) Sanière, L.; Leman, L.; Bourguignon, J.-J.; Dauban, P.; Dodd, R. H. Tetrahedron 2004, 60, 5889.

(14) Benohoud, M.; Leman, L.; Cardoso, S. H.; Retailleau, P.; Dauban, P.; Thierry, J.; Dodd, R. H. J. Org. Chem. 2009, 74, 5331.

(15) Daniel, M.; Blanchard, F.; Nocquet-Thibault, S.; Cariou, K.; Dodd, R. H. J. Org Chem. 2015, 80, 10624.

(16) Wuts, P. G. M.; Greene T.W. Greene's Protective Groups in Organic Synthesis, Fourth Edition, John Wiley \&Sons : Hoboken, 2006.

(17) (a) Albrecht, C.; Barnes, S.; Böckemeier, H.; Davies, D.; Dennis, M.; Evans, D. M.; Fletcher, M. D.; Jones, I.; Leitmann, V.; Murphy, P. J.; Rowles, R.; Nash, R.; Stephenson, R. A.; Horton P. N.; Hursthouse, M. B. Tetrahedron Lett. 2008, 49, 185. (b) Davies, D.; Fletcher, M. D.; Franken, H.; Hollinshead, J.; Kähm, K.; Murphy, P. J.; Nash, R.; Potter, D. M. Tetrahedron Lett. 2010, 51, 6825. (c) Al Shuhaib, Z.; Davies, D. H.; Dennis, M.; Evans, D. M.; Fletcher, M. D.; Franken, H.; Hancock, P.; Hollinshead, J.; Jones, I.; Kähm, K.; Murphy, P. J.; Nash, R.; Potter, D.; Rowles, R. Tetrahedron 2014, 70, 4412.

(18) Cobb, S. L. ; Vederas, J. C. Org. Biomol. Chem. 2007, 5, 1031.

(19) Baldwin, J. E. ; Spivey, A. C. ; Schofield, C. J. Tetrahedron Asymmetry 1990, 1, 881.

(20) Methyl (2S)-3-(2,3-bis(benzyloxycarbonyl)guanidino)-2-( $p$ methoxybenzylamino) propanoate (14): To a solution of amine 12 (669 mg, $2.8 \mathrm{mmol}$ ) in $\mathrm{CH}_{2} \mathrm{Cl}_{2}(15 \mathrm{~mL}$ ) were successively added at room temperature 1,3-bis(benzyloxycarbonyl)-2methylisothiourea $(\mathbf{1 3}, 1.22 \mathrm{~g}, 3.4 \mathrm{mmol})$ and triethylamine $(0.93$ $\mathrm{mL}, 6.7 \mathrm{mmol}$ ). The reaction mixture was stirred overnight, the solvent was removed under vacuum and the residue was purified by flash column chromatography on silica gel (EtOAc/heptane, 40:60), providing compound 14 as a yellow oil ( $1.47 \mathrm{~g}, 95 \%$ yield from 11). $[\alpha]_{\mathrm{D}^{20}}+5.46\left(\mathrm{c}=0.97, \mathrm{CHCl}_{3}\right)$; IR (neat, $\left.\mathrm{cm}^{-1}\right): v_{\max } 3330$, 1733, 1638, 1561, 1513, 1246, 1204, 1054, 698; ${ }^{1} \mathrm{H}$ NMR $(300$ $\mathrm{MHz}_{\mathrm{CDCl}}$ ): $\delta 11.7(\mathrm{~s}, 1 \mathrm{H}, \mathrm{NH}), 8.82$, (br s, $\left.1 \mathrm{H}, \mathrm{NH}\right), 7.37(\mathrm{~m}, 6 \mathrm{H}$, $\mathrm{CH}, \mathrm{Cbz}$ ), 7.30 (d, J = 7.5 Hz, 4H, Cbz), 7.26 (d, J=8.6 Hz, 2H, CH, PMB), 6.80 (d, $J=8.6 \mathrm{~Hz}, 2 \mathrm{H}, \mathrm{CH}$ PMB), 5.19 (d, $J=1.7 \mathrm{~Hz}, 2 \mathrm{H}, \mathrm{CH}_{2}$, Cbz), 5.09 (s, 2H, CH2), 3.90-3.79 (m, 1H), 3.80 (d, J = $12.8 \mathrm{~Hz}, 1 \mathrm{H}$, $\mathrm{CH}_{2}, \mathrm{PMB}$ ), 3.76 (s, 3H, MeO), 3.70 (s, 3H, MeO), 3.64 (d, $J=12.8$ $\mathrm{Hz}, 1 \mathrm{H}, \mathrm{CH}_{2}$, PMB), 3.64-3.37 (m, 2H), 1.85 (br s, $1 \mathrm{H}, \mathrm{NH}$ ); ${ }^{13} \mathrm{C} \mathrm{NMR}$ (75 MHz, $\mathrm{CDCl}_{3}$ ): $\delta 173.4(\mathrm{C}), 163.8(\mathrm{C}), 159.0(\mathrm{C}), 156.2(\mathrm{C}), 153.7$ (C), 136.9 (C), 134.9 (C), 131.4 (C), 129.8 (2 CH, PMB), $129.0(\mathrm{CH}$, Cbz), 128.9 (CH, Cbz), 128.8 (CH, Cbz), 128.6 (CH, Cbz), $128.4(\mathrm{CH}$, Cbz), 128.1 (CH, Cbz), 114.0 (2 CH, PMB), $68.4\left(\mathrm{CH}_{2}, \mathrm{Cbz}\right), 67.3$ $\left(\mathrm{CH}_{2}, \mathrm{Cbz}\right), 58.8(\mathrm{CH}-\mathrm{N}), 55.5\left(\mathrm{CH}_{3}, \mathrm{MeO}\right), 52.5\left(\mathrm{CH}_{3}, \mathrm{MeO}\right), 51.4$ $\left(\mathrm{CH}_{2}\right), 42.8\left(\mathrm{CH}_{2}\right)$. HRMS $\mathrm{m} / z$ calcd for $\mathrm{C}_{29} \mathrm{H}_{33} \mathrm{~N}_{4} \mathrm{O}_{7}[\mathrm{M}+\mathrm{H}]^{+}$: 549.2344; found: 549.2329 .

$N$-Methoxy- $N$-methyl (2S)-3-(2,3-bis(benzyloxycarbonyl)guanidino)-2-( $p$-methoxybenzylamino)propanamide (27): Treatment of amine 25 ( $0.7 \mathrm{mmol})$ as described above provided, after flash chromatography of the crude product on silica gel (EtOAc/heptane, 6:4), compound 26 as a yellow oil (87\% yield from 24). $[\alpha]_{\mathrm{D}^{20}}-15.27$ ( $\mathrm{c}=1.12, \mathrm{CHCl}_{3}$ ); IR (neat, $\mathrm{cm}^{-1}$ ): $v_{\max }$ 3330, 2931, 1732, 1638, 1563, 1512, 1245, 1204, 1052, 803, 746, 697; ${ }^{1} \mathrm{H}$ NMR (300 MHz, $\mathrm{CDCl}_{3}$ ): $\delta 11.6$ (br s, $1 \mathrm{H}, \mathrm{NH}$ ), 8.85, (br s, $1 \mathrm{H}, \mathrm{NH}), 7.40-7.24(\mathrm{~m}, 10 \mathrm{H}, \mathrm{CH}, \mathrm{Cbz}) 7.28(\mathrm{~d}, J=8.6 \mathrm{~Hz}, 2 \mathrm{H}, \mathrm{CH}$, PMB), 6.78 (d, $J=8.6 \mathrm{~Hz}, 2 \mathrm{H}, \mathrm{CH}, \mathrm{PMB}), 5.24(\mathrm{~d}, J=12.2 \mathrm{~Hz}, 1 \mathrm{H}$, $\left.\mathrm{CH}_{2}, \mathrm{Cbz}\right), 5.18\left(\mathrm{~d}, J=12.2 \mathrm{~Hz}, 1 \mathrm{H}, \mathrm{CH}_{2}, \mathrm{Cbz}\right), 5.10(\mathrm{~d}, J=12.4 \mathrm{~Hz}$, $1 \mathrm{H}, \mathrm{CH}_{2}$ ), $5.05\left(\mathrm{~d}, J=12.4 \mathrm{~Hz}, 1 \mathrm{H}, \mathrm{CH}_{2}\right), 3.89-3.81\left(\mathrm{~m}, 1 \mathrm{H}, \mathrm{CH}_{2}\right)$, 
3.79 (d, $\left.J=13.0 \mathrm{~Hz}, 1 \mathrm{H}, \mathrm{CH}_{2}, \mathrm{PMB}\right), 3.75$ (s, 3H, MeO), 3.74-3.70 (m, $1 \mathrm{H}, \mathrm{CH}), 3.54(\mathrm{~s}, 3 \mathrm{H}, \mathrm{MeO}), 3.51\left(\mathrm{~d}, J=12.8 \mathrm{~Hz}, 1 \mathrm{H}, \mathrm{CH}_{2}, \mathrm{PMB}\right)$ 3.15 (s, 3H, MeN), 3.20-3.10 (m, 1H, $\mathrm{CH}_{2}$ ), $1.96(\mathrm{br} \mathrm{s}, 1 \mathrm{H}, \mathrm{NH}) ;{ }^{13} \mathrm{C}$ NMR (75 MHz, CDCl 3 ): $\delta 163.8$ (C), 158.9 (C), 156.1 (C), 153.7 (C), 137.0 (C), 135.0 (C), 131.8 (C), 130.4 (2 CH, PMB), 128.9 (CH, Cbz), 128.9 (CH, Cbz), 128.8 (CH, Cbz), 128.6 (CH, Cbz), 128.3 (CH Cbz), 128.1 (CH Cbz), 113.9 (2 CH PMB), $68.3\left(\mathrm{CH}_{2}, \mathrm{Cbz}\right), 67.2\left(\mathrm{CH}_{2}\right.$, $\mathrm{Cbz}), 61.8\left(\mathrm{CH}_{3} \mathrm{MeO}\right), 55.7(\mathrm{CH}-\mathrm{N}), 55.5\left(\mathrm{CH}_{3}, \mathrm{MeO}\right), 51.4\left(\mathrm{CH}_{2}\right)$, $43.3\left(\mathrm{CH}_{2}\right), 32.5\left(\mathrm{CH}_{3}, \mathrm{MeN}\right)$. HRMS $\mathrm{m} / z$ calcd for $\mathrm{C}_{30} \mathrm{H}_{36} \mathrm{~N}_{5} \mathrm{O}_{7}$ $[\mathrm{M}+\mathrm{H}]^{+}: 578.2609$; found: 578.2618 .

(21) Methyl (4S)-2-(benzyloxycarbonylimino)-3-(p-methoxybenzyl)-imidazoledine-4-carboxylate (15): A solution of compound $14(2.56 \mathrm{~g}, 4.6 \mathrm{mmol})$ in acetonitrile $(25 \mathrm{~mL})$ was heated at $70{ }^{\circ} \mathrm{C}$ for $2 \mathrm{~d}$. The reaction mixture was then cooled to room temperature, the solvent was removed under vacuum and the residue was purified by flash column chromatography on silica gel (EtOAc/heptane, 1:1) to provide the cyclic guanidine $\mathbf{1 5}$ as a colorless oil $(1.39 \mathrm{~g}, 75 \%)$. [ $\alpha]_{\mathrm{D}}{ }^{20}+25.67\left(\mathrm{c}=0.9, \mathrm{CHCl}_{3}\right)$; IR (neat, $\mathrm{cm}^{-1}$ ): $v_{\max } 3374,2926,1646,1587,1513,1248,1128$, 1095, 799; ${ }^{1} \mathrm{H}$ NMR (300 MHz, CDCl $): ~ \delta 7.95$, (br s, $\left.1 \mathrm{H}, \mathrm{NH}\right), 7.37$ $(\mathrm{d}, J=6.8 \mathrm{~Hz}, 2 \mathrm{H}, \mathrm{Cbz}), 7.31-7.21(\mathrm{~m}, 3 \mathrm{H}, \mathrm{Cbz}), 7.10(\mathrm{~d}, J=8.6 \mathrm{~Hz}$, $2 \mathrm{H}, \mathrm{CH}, \mathrm{PMB}), 6.78$ (d, $J=8.6 \mathrm{~Hz}, 2 \mathrm{H}, \mathrm{CH}$ PMB), 5.16-5.05 (m, 3H), 4.10-4.03 (m, 2H,), $3.99\left(\mathrm{dd}, J=10.0,6.0 \mathrm{~Hz}, 1 \mathrm{H}, \mathrm{CH}_{2}\right), 3.73(\mathrm{~s}, 3 \mathrm{H}$, $\mathrm{MeO}$ ), 3.67 (s, 3H, MeO), $3.63\left(\mathrm{dd}, J=10.0,6.0 \mathrm{~Hz}, 1 \mathrm{H}, \mathrm{CH}_{2}\right) ;{ }^{13} \mathrm{C}$ NMR (75 MHz, CDCl 3 ): $\delta 170.4(\mathrm{C}), 164.4(\mathrm{C}), 163.5$ (C), 159.3 (C), 137.3 (C), 130.0 (2 CH, PMB), 128.3 (2 CH, Cbz), 128.2 (2 CH, Cbz),
127.7 (CH, Cbz), 114.1 (2 CH, PMB), $67.0\left(\mathrm{CH}_{2}, \mathrm{Cbz}\right), 56.4(\mathrm{CH}-\mathrm{N})$, $55.3\left(\mathrm{CH}_{3}, \mathrm{MeO}\right), 52.7\left(\mathrm{CH}_{3}, \mathrm{MeO}\right), 46.2\left(\mathrm{CH}_{2}\right), 44.5\left(\mathrm{CH}_{2}\right)$. HRMS $\mathrm{m} / z$ calcd for $\mathrm{C}_{21} \mathrm{H}_{24} \mathrm{~N}_{3} \mathrm{O}_{5}[\mathrm{M}+\mathrm{H}]^{+}: 398.1710$; found: 398.1711 .

$N$-Methoxy- $N$-methyl (4S)-2-(benzyloxycarbonylimino)-3-( $p$ methoxybenzyl)imidazolidine-4-carboxamide (28). Treatment of compound $26(0.6 \mathrm{mmol})$ as described above provided, after flash column chromatography of the crude product on silica gel (EtOAc/heptane, 8:2), compound $\mathbf{2 7}$ as a colorless oil $(74 \%)$. $[\alpha]_{\mathrm{D}}{ }^{20}$ $+27.39\left(\mathrm{c}=0.92, \mathrm{CHCl}_{3}\right.$ ); IR (neat, $\left.\mathrm{cm}^{-1}\right): v_{\max } 3374,2926,1646$, $1587,1513,1248,1128,1095,799 ;{ }^{1} \mathrm{H}$ NMR $\left(300 \mathrm{MHz}, \mathrm{CDCl}_{3}\right): \delta$ 7.94, (br s, $1 \mathrm{H}, \mathrm{NH}), 7.43-7.24$ (m, 5H, CH, Cbz), 7.26 (d, $J=8.5 \mathrm{~Hz}$, 2H, CH, PMB), 6.81 (d, $J=8.5 \mathrm{~Hz}, 2 \mathrm{H}, \mathrm{CH}, \mathrm{PMB}), 5.23$ (d, $J=14.7 \mathrm{~Hz}$, $1 \mathrm{H}, \mathrm{CH}_{2}$ ), 5.16 (d, $J=12.4,2 \mathrm{H}, \mathrm{CH}_{2}$ ), $5.10\left(\mathrm{~d}, J=12.4,2 \mathrm{H}, \mathrm{CH}_{2}\right), 4.27$ (dd, $J=10.6 \mathrm{~Hz}, 6.5 \mathrm{~Hz}, 1 \mathrm{H}, \mathrm{CH}), 3.98\left(\mathrm{~d}, J=14.7,1 \mathrm{H}, \mathrm{CH}_{2}\right), 3.81-3.73$ (m, $1 \mathrm{H}, \mathrm{CH}_{2}$ ), 3.76 (s, 3H, MeO), 3.46 (dd, J = $9.6 \mathrm{~Hz}, 6.6 \mathrm{~Hz}, 1 \mathrm{H}, \mathrm{CH}_{2}$ ), $3.35(\mathrm{~s}, 3 \mathrm{H}, \mathrm{MeO}), 3.14(\mathrm{~s}, 3 \mathrm{H}, \mathrm{MeN}) ;{ }^{13} \mathrm{C}$ NMR $\left(75 \mathrm{MHz}, \mathrm{CDCl}_{3}\right): \delta$ 169.9 (C), 164.6 (C), 164.2 (C), 159.4 (C), 137.6 (C), 130.4 (2 CH, PMB), 128.5 (CH, Cbz), 128.3 (CH, Cbz), 127.8 (CH, Cbz), 114.2 (2 CH, PMB), $67.1\left(\mathrm{CH}_{2}, \mathrm{Cbz}\right), 61.4\left(\mathrm{CH}_{3}, \mathrm{MeO}\right), 55.5\left(\mathrm{CH}_{3}, \mathrm{Me}\right)$ ), 55.0 ( $\mathrm{CH}-$ N), $45.9\left(\mathrm{CH}_{2}\right), 44.6\left(\mathrm{CH}_{2}\right), 29.9\left(\mathrm{CH}_{3}, \mathrm{MeN}\right)$. HRMS $m / z$ calcd for $\mathrm{C}_{22} \mathrm{H}_{27} \mathrm{~N}_{4} \mathrm{O}_{5}[\mathrm{M}+\mathrm{H}]^{+}: 427.1976$; found: 427.1984 . 